\title{
Use of medical expulsive therapy in children: An assessment of nationwide practice patterns and outcomes
}

\author{
Jonathan S. Ellison ${ }^{\mathrm{a}}$, Paul A. Merguerian ${ }^{\mathrm{a}}$, Benjamin C. Fu ${ }^{\mathrm{a}}$, Sarah K. Holt ${ }^{\mathrm{b}}$, Thomas S. \\ Lendvay $^{\mathrm{a}}$, John L. Gore ${ }^{\mathrm{b}}$, and Margarett Shnorhavorian ${ }^{\mathrm{a}}$ \\ aDivision of Pediatric Urology, Seattle Children's Hospital, Seattle, WA, USA \\ ${ }^{\mathrm{b}}$ Department of Urology, University of Washington, Seattle, WA, USA
}

\section{Summary}

Introduction-Early data support the use of medical expulsive therapy (MET) in children. However, little is known regarding use or outcomes associated with MET outside of pediatricspecific practices. Using a national administrative dataset, we sought to characterize utilization patterns of MET as well as assess outcomes associated with MET exposure.

\begin{abstract}
Study design-We interrogated the MarketScan Commercial Claims and Encounters database to identify children under the age of 18 presenting to the emergency department (ED) with any diagnosis of upper urinary tract calculi (UUTC, including renal and ureteral calculi). MET exposure was defined as having a prescription filled for a MET agent within 1 week of the ED encounter. Characteristics of children receiving MET were defined and outcomes compared between children with and without MET exposure.
\end{abstract}

Results-Of 1325 children included in the study, 13.2\% received MET, including $15.4 \%$ of children with a diagnosis of "calculus of the ureter." MET use increased significantly throughout the study period $(p=0.004)$, although only $30.4 \%$ of children considered potential MET candidates received MET in the final year of the study (2013). Among all patients, receipt of MET was associated with male gender, presence of comorbidity, provider-type (urologist), and year of diagnosis, although among those with a specific diagnosis of "calculus of the ureter," only year of diagnosis remained a significant factor. Rates of unplanned physician visits and surgical interventions were similar between groups. Children receiving MET were more likely to receive follow-up imaging, although only $46 \%$ of children with ureteral calculi had appropriate follow-up imaging within 90 days, regardless of MET exposure. Odds ratios of factors and outcomes associated with MET exposure are shown in the Table.

Discussion-Although early data support safety and efficacy MET in children, nationwide use in children is low among potential candidates for MET. For children with ureteral calculi, only year of diagnosis was a significant factor associated with MET use. No difference in unplanned

Correspondence to: Jonathan S. Ellison, Seattle Children's Hospital, Urology, OA 9.220, 4800 Sand Point Way, Seattle, WA 98119, USA, Tel.: +1 206987 0155; fax: +1 206469 0774, Jonathan.ellison@ seattlechildrens.org (J.S. Ellison).

Conflict of interest

Dr. Lendvay is CMO, co-founder, board member, and equity owner of CSATS Inc. This interest does not have any conflict with the current research. No other authors have any interests to disclose. 
physician visits or surgical interventions was noted. Most notable, however, was the low rate of follow-up imaging within 90 days for children presenting acutely with UUTC.

Conclusions-Use of MET for children with ureteral calculi is increasing, although still fewer than a third of children considered potential candidates receive this treatment. Follow-up imaging is not obtained for many children with ureteral calculi. Future work is needed to standardize management and follow-up protocols for children with acute renal colic.

\section{Keywords}

Ureteral calculi; Kidney calculi; Pediatrics; Tamsulosin

\section{Introduction}

The incidence of pediatric upper urinary tract calculi (UUTC) has doubled within the past decade, and is now estimated at 3.3\% [1-4]. As ureteral stones pass spontaneously in 41$63 \%$ of children, many children with acute renal colic can be managed without hospital admission $[5,6]$. However, $22 \%$ of these children may require surgery for UUTC, with $25 \%$ needing more than one operation, underscoring the importance of minimizing surgical intervention in these children [7].

Although the efficacy of medical expulsive therapy (MET) is established in adults, use is provider dependent and not widespread [8-10]. Efficacy of MET has also been supported in children, including a recent meta-analysis showing improved passage rates with this treatment [11-13]. While MET use in children appears to be increasing at pediatric hospitals, little is known regarding specific practice patterns of MET in children with urolithiasis [1].

Using a commercial medical claims database, we assessed the use of medical expulsive therapy in children presenting to the emergency department (ED) with UUTC. Our study had two aims: first, to characterize patient and provider-level factors associated with MET use in children; and second, to evaluate outcomes of patients receiving MET, hypothesizing that MET would result in lower rates of unplanned ED visits and need for surgical intervention.

\section{Methods}

\section{Data source}

For this study, we used the Thomson Reuters MarketScan Commercial Claims and Encounters database from 2007 to 2013. Overall the MarketScan claims databases have 143 million unique patients including on 50 million covered lives in the most recent full data year. The sample includes Americans with employer-provided health insurance and Medicaid, although the majority of MarketScan data are sourced from large employers. The dataset captures longitudinal billing and claims data on inpatient admissions, ED evaluations, and outpatient visits and employer-provided data also allows tracking of patients across health plans enabling capture of patients who would be otherwise "lost" in plan-based data sources [14]. 


\section{Study population}

We identified children 1-18 years of age presenting to the ED with a diagnosis of UUTC, including renal and ureteral calculi, using International Classification of Disease (ICD-09) codes 592.0, 592.1, 592.9, or 274.11. Children had to have continuous benefits coverage for a minimum of 90 days prior to and 120 days following the ED evaluation for UUTC. Children were excluded if they had received a surgical intervention (primary therapy or diversion with a ureteral stent or percutaneous nephrostomy) at the time of or within 30 days prior to the ED encounter. To ensure only selection of children considered potential candidates for MET, children were also excluded if they had an active urinary tract infection (ICD-09 codes 599.0, 590.0, and 590.1) or acute renal insufficiency (ICD-09 codes 584.0, 584.5, 584.9, and 586) at the time of evaluation, both contraindications for MET. MET exposure was defined as prescription filled within 1 week of the initial encounter date for tamsulosin, doxazosin, terazosin, alfuzosin, or nifedipine. A subset analysis was performed for children with the ICD-09 code specific for "calculus of the ureter" (ICD-09 code 592.1) to evaluate those children for whom MET would be specifically indicated. For this subanalysis, children with "calculus of the ureter" plus any other codes specific to UUTC were included in the "calculus of the ureter" group.

\section{Patient and provider characteristics}

Children were categorized based on age at time of evaluation, gender, geographic region, and insurance status. Comorbidity was defined as a binary variable with presence or absence of a chronic comorbid condition as described by Feudnter et al. [15]. Provider type at encounter was also characterized.

\section{Outcomes}

Outcomes measures were collected within 90 days of the initial ED encounter. Primary outcome measure was surgical intervention within 90 days of ED encounter. Secondary outcomes included provider encounters through the ED, inpatient admission or outpatient visits, need for surgical intervention, and use of follow-up imaging. Appropriate follow-up imaging was defined as ultrasound (US) or plain film of the kidney-ureter-bladder (KUB) [16].

\section{Statistical analyses}

Univariate comparisons of demographic, provider and MET exposure measures for children with a specific ureteral calculus versus children with all other diagnoses of UUTC were done using the Student's $t$ test for continuous variables and the chi-square test for categorical variables. Logistic regression was used to assess demographic characteristics associated with MET for both unadjusted and adjusted risk estimates in the overall study population, as well as among the subset of children with ureteral calculus.

Each outcome had a separate model built with MET use as the primary exposure. The associations between outcome measures and MET use were calculated using multivariate logistic regression adjusted for age, sex, urologist provider, presence of complex chronic condition, and year of diagnosis. The Hosmer-Lemeshow statistic was used to evaluate 
model goodness-of-fit. All tests of significance were two-tailed and set at a $p$-value of $<0.05$. All analyses were performed using SAS 9.3 (SAS Institute Inc., Cary, NC, USA).

\section{Results}

A total of 2616 children presented to the ED with a diagnosis of UUTC during the study period. Of these, 691 were excluded, leaving 1325 children included in our analysis (Fig. 1).

Of the total group of children with UUTC, 175 (13.2\%) received MET. Five-hundred and twenty-seven children had a diagnosis of "calculus of the ureter," of whom $81(15.2 \%)$ received MET. As seen in Fig. 2, MET use increased significantly over time from $12.9 \%$ in 2008 to $30.4 \%$ in 2013 ( $p<0.001)$. Demographic information of the entire group and those with "calculus of the ureter" are noted in Table 1. Children with a diagnosis of "calculus of the ureter" had a lower proportion of comorbidity and were more often evaluated by a urologist during their ED visit.

Factors associated with MET exposure are displayed in Table 2. While multiple factors were associated with increased odds of MET receipt in the entire group, only year of exposure was significantly associated with MET exposure in the "calculus of the ureter" group.

Outcomes for the first 90 days following the initial ED visit are displayed in Table 3. All models showed a good fit with the data $(p>0.05)$. No significant differences were noted in unplanned ED visits or receipt of surgical procedure between groups. Appropriate imaging follow-up was more likely in the MET group regardless of diagnosis classification. Lack of any follow-up imaging was seen in $48 \%$ and $43 \%$ of the entire group and "calculus of the ureter" sub-group, respectively. Of those patients receiving followup imaging, computed tomography (CT) was obtained in $24 \%$.

\section{Discussion}

Overall use of MET in children presenting to the ED with UUTC in this database was low. While use of MET increased significantly throughout the study period, just under one-third of children considered candidates for MET received therapy at the end of the study period. Gender and provider specialty were associated with variation in MET use in all diagnoses, but not in the sub-group of patients with ureteral calculus. Physician visits and surgical interventions were not associated with differences in MET use. Appropriate follow-up imaging studies were not obtained in a large proportion of patients (54.1\% overall), although MET was associated with increased odds of appropriate follow-up imaging.

A continued rise in ED discharges in contrast to a decline of inpatient admissions for pediatric UUTC emphasizes the need for optimal outpatient management [17]. A recent review by Hernandez et al. argued that acute management of pediatric UUTC can be accomplished while avoiding hospital admission, further emphasizing the importance of optimizing outpatient management [18]. Recent studies have supported the efficacy of MET in the pediatric population [13]. Over the entire study period, only $15 \%$ of children considered potential candidates for MET received a prescription for a MET agent, remarkably similar to a $17 \%$ utilization rate of MET published in the adult literature [10]. 
Interestingly, MET use increased significantly over the study period, perhaps because of new literature supporting MET safety and efficacy in children [12,19]. A similar degree of increase in MET use was noted over a 5 year study period in the adult population, from 2002 to 2006 [20]. Thus, the increase in MET use for pediatric UUTC likely represents a continuous trend of widespread dissemination of expulsive therapy as a treatment option for ureteral calculi in a variety of patient populations. While gender and provider type also appeared to be associated with MET exposure in the entire group, these associations were not seen in the specific ureteral calculi group. Whether this is because of lower numbers in this group thus increasing the likelihood of a type II error, or because of a truly different group owing to the increased specificity of this coding group is unclear. However, it is remarkable that MET exposure was similar in both the ureteral calculus subgroup and the entire cohort. Notably, medically expulsive therapy is not an approved indication for alphablockers or calcium-channel blockers according to the Food and Drug Administration, and providers or parents may not feel comfortable with the use of an "off-label" medication in this population. Furthermore, providers not familiar with the safety and efficacy of MET agents, specifically tamsulosin, in children may be less likely to offer this treatment option. Other explanations for low use of MET in patients with ureteral calculi could include stone size or location within the ureter, both of which have been shown to influence stone passage rates [21]. It is notable that no patients under the age of 5 received this therapy and may indicate provider concerns regarding the ability of young patients to tolerate this medication, although previous studies on MET have included patients younger than 5 [12,19,22]. Finally, while concomitant urological diagnoses are not specific contra-indications for MET, the presence of these diagnoses could influence provider decision making and were not assessed in this study.

Children receiving MET did not have lower rates of unexpected physician encounters or surgical intervention. While the vast majority of children had outpatient follow-up, only 52\% of the entire cohort and 57\% of those with a diagnosis of "calculus of the ureter" received any imaging in follow-up, with MET exposure being associated with an increased odds of receiving appropriate follow-up imaging. Follow-up imaging is recommended for children with ureteral calculi to assess both stone location and collecting system dilatation, unless a "stone in hand" is available to confirm passage [16]. While US and KUB were the most commonly obtained follow-up imaging studies, $24 \%$ of children with follow-up imaging received a CT scan instead of the preferred follow-up studies. Overuse of CT scan in the initial diagnosis of UUTC has been well described $[23,24]$ while reduction of ionizing radiation has been highlighted as a quality of care issue in pediatrics [25] As such, further investigation is warranted into follow-up imaging practices for children with UUTC, regarding both timing and modality.

Our study has several limitations. We rely on physician-based diagnostic codes to identify potential candidates for MET. The code for "nephrolithiasis", however, may include children with both ureteral and renal calculi, limiting conclusions from this larger group. The specificity of "calculus of the ureter" to identify children with strictly ureteral calculi has been previously reported in other administrative databases at 100\% [26]. Thus, sub-analysis of those children with the specific diagnostic code 592.1, while limited in number, provides a more accurate comparison for children considered to be candidates for MET. Furthermore, 
factors that might influence decision for MET such as stone size and location, patient comorbidity, previous response to MET, and pain at time of diagnosis are not available in the MarketScan dataset. Thus, while all included children with a diagnosis of "calculus of the ureter" were considered candidates for MET, some children may have been clinically determined poor candidates based on variables not available within the dataset. As such, an assessment of comparative-effectiveness between MET and expectant management for ureteral calculi in children cannot be made from these data. MET exposure was based on filled-prescriptions within 7 days of ED visits and assessment of adherence to the medication cannot be assessed. Poor adherence to MET could contribute to the lack of significant differences in outcomes between both groups. However, assessment of patient compliance has been limited, even in prospective randomized-controlled trials [21]. In addition, this situation represents a "real-world" situation with regards to patient adherence. Finally, our study included only privately insured patients with continuous coverage enrollment and generalizability to other populations may be limited.

These limitations notwithstanding, our study has several important implications for the management of children with UUTC. First, although MET utilization in children considered potential candidates increased over the entire study period, use of this therapy is still quite low. Unmeasured variables, such as stone size and location, may have led providers to determine that the child was a poor candidate for MET. However, even acknowledging these factors, it seems unlikely that only $15 \%$ of children would have been deemed an appropriate candidate for expulsive therapy. While evolving familiarity with this treatment among medical providers may explain the increased utilization patterns, further work is needed in selecting the most appropriate candidates for MET. Second, we found a surprisingly low proportion of children presenting with ureteral calculi receiving confirmatory follow-up imaging. This finding is of concern, as documentation of a passed ureteral calculus is important to prevent long-term sequellae of ureteral obstruction. As MarketScan is a payer database capable of capturing individual healthcare events at multiple locations, it is unlikely that significant numbers of follow-up imaging were missed in our analysis. Furthermore, a surprisingly high number of children received CT scan at follow-up, raising concerns regarding radiation-exposure in this population. Lastly, while comparative-effectiveness of MET is limited, as discussed above, it is notable that there was no difference in ultimate need for surgical intervention among groups. Previous reports have indicated that $25 \%$ of children presenting acutely with UUTC will proceed to surgical intervention within 6 months, consistent with our findings [7]. Notably, the SUSPEND (Spontaneous Urinary Passage ENabled by Drugs) trial recently published found no benefit for MET in adults, reinvigorating a debate about MET in the adult population [21]. This study could not evaluate time for stone passage or proportion of successful stone passages, outcomes for which MET has been shown to improve. However, our study evaluated health-service related outcomes such as physician visits and receipt of surgery as stone-passage rates cannot be abstracted from the MarketScan dataset. Notably, these endpoints were not explored in other trials on MET in children [12,19,22], and represent opportunities for further study regarding efficacy of MET in children. 


\title{
Conclusions
}

MET is a therapeutic option for children with ureteral calculi [27]. Our data suggest that a minority of children considered to be candidates for MET receive this therapy. However, MET use has increased over time. Many children with ureteral calculi do not receive followup imaging within 90 days of diagnosis, and further investigation to follow-up patterns in children with ureteral calculi is warranted. Outcomes did not differ based on MET exposure in our study, although broader conclusions are limited potential unmeasured confounding variables. Large-scale randomized studies are needed to further define the role of MET in children with ureteral calculi.

\section{Acknowledgments}

\author{
Funding \\ None.
}

\section{References}

1. Bush NC, Xu L, Brown BJ, Holzer MS, Gingrich A, Schuler B, et al. Hospitalizations for pediatric stone disease in United States, 2002-2007. J Urol. 2010; 183:1151-6. [PubMed: 20096871]

2. Dwyer ME, Krambeck AE, Bergstralh EJ, Milliner DS, Lieske JC, Rule AD. Temporal trends in incidence of kidney stones among children: a 25-year population based study. J Urol. 2012; 188:247-52. [PubMed: 22595060]

3. Sas DJ, Hulsey TC, Shatat IF, Orak JK. Increasing incidence of kidney stones in children evaluated in the emergency department. J Pediatr. 2010; 157:132-7. [PubMed: 20362300]

4. Tasian GE, Ross ME, Song L, Sas DJ, Keren R, Denburg MR, et al. Annual incidence of nephrolithiasis among children and adults in South Carolina from 1997 to 2012. Clin J Am Soc Nephrol. 2016; 11(3):488-96. [PubMed: 26769765]

5. Pietrow PK, Pope JCt, Adams MC, Shyr Y, Brock JW 3rd. Clinical outcome of pediatric stone disease. J Urol. 2002; 167:670-3. [PubMed: 11792950]

6. Kalorin CM, Zabinski A, Okpareke I, White M, Kogan BA. Pediatric urinary stone disease-does age matter? J Urol. 2009; 181:2267-71. discussion 71. [PubMed: 19296968]

7. Routh JC, Graham DA, Nelson CP. Trends in imaging and surgical management of pediatric urolithiasis at American pediatric hospitals. J Urol. 2010; 184:1816-22. [PubMed: 20728146]

8. Hollingsworth JM, Rogers MA, Kaufman SR, Bradford TJ, Saint S, Wei JT, et al. Medical therapy to facilitate urinary stone passage: a meta-analysis. Lancet. 2006; 368:1171-9. [PubMed: 17011944]

9. Hollingsworth JM, Davis MM, West BT, Wolf JS Jr, Hollenbeck BK. Trends in medical expulsive therapy use for urinary stone disease in U.S. Emergency Departments. Urology. 2009; 74:1206-9. [PubMed: 19811807]

10. Scales CD, Bergman J, Carter S, Jack G, Saigal CS, Litwin MS. Quality of acute care for patients with urinary stones in the United States. Urology. 2015; 86(5):914-21. [PubMed: 26335495]

11. Tasian GE, Cost NG, Granberg CF, Pulido JE, Rivera M, Schwen Z, et al. Tamsulosin and spontaneous passage of ureteral stones in children: a multi-institutional cohort study. J Urol. 2014; 192:506-11. [PubMed: 24518765]

12. Mokhless I, Zahran AR, Youssif M, Fahmy A. Tamsulosin for the management of distal ureteral stones in children: a prospective randomized study. J Pediatr Urol. 2012; 8:544-8. [PubMed: 22099477]

13. Velazquez N, Zapata D, Wang HS, Wiener JS, Lipkin ME, Routh JC. Medical expulsive therapy for pediatric urolithiasis: systematic review and meta-analysis. J Pediatr Urol. 2015; 11(6):321-7. [PubMed: 26165192] 
14. Hansen L, Chang S. Health research data for the real world: the MarketScan databases. White paper. Jul.2012:2013.

15. Feudtner C, Christakis DA, Connell FA. Pediatric deaths attributable to complex chronic conditions: a population-based study of Washington State, 1980-1997. Pediatrics. 2000; 106:2059. [PubMed: 10888693]

16. Fulgham PF, Assimos DG, Pearle MS, Preminger GM. Clinical effectiveness protocols for imaging in the management of ureteral calculous disease: AUA technology assessment. J Urol. 2013; 189:1203-13. [PubMed: 23085059]

17. Kusumi K, Becknell B, Schwaderer A. Trends in pediatric urolithiasis: patient characteristics, associated diagnoses, and financial burden. Pediatr Nephrol. 2015; 30:805-10. [PubMed: 25481020]

18. Hernandez JD, Ellison JS, Lendvay TS. Current trends, evaluation, and management of pediatric nephrolithiasis. JAMA Pediatr. 2015; 169:964-70. [PubMed: 26302045]

19. Aydogdu O, Burgu B, Gucuk A, Suer E, Soygur T. Effectiveness of doxazosin in treatment of distal ureteral stones in children. J Urol. 2009; 182:2880-4. [PubMed: 19846149]

20. Hollingsworth JM, Wolf JS Jr, Faerber GJ, Roberts WW, Dunn RL, Hollenbeck BK. Understanding the barriers to the dissemination of medical expulsive therapy. J Urol. 2010; 184:2368-72. [PubMed: 20952037]

21. Pickard R, Starr K, MacLennan G, Lam T, Thomas R, Burr J, et al. Medical expulsive therapy in adults with ureteric colic: a multicentre, randomised, placebo-controlled trial. Lancet. 2015; 386:341-9. [PubMed: 25998582]

22. Erturhan S, Bayrak O, Sarica K, Seckiner I, Baturu M, Sen H. Efficacy of medical expulsive treatment with doxazosin in pediatric patients. Urology. 2013; 81:640-3. [PubMed: 23343614]

23. Tasian GE, Pulido JE, Keren R, Dick AW, Setodji CM, Hanley JM, et al. Use of and regional variation in initial CT imaging for kidney stones. Pediatrics. 2014; 134:909-15. [PubMed: 25349323]

24. Johnson EK, Graham DA, Chow JS, Nelson CP. Nationwide emergency department imaging practices for pediatric urolithiasis: room for improvement. J Urol. 2014; 192:200-6. [PubMed: 24518772]

25. Stratton KL, Pope JCt, Adams MC, Brock JW 3rd, Thomas JC. Implications of ionizing radiation in the pediatric urology patient. J Urol. 2010; 183:2137-42. [PubMed: 20399463]

26. Semins MJ, Trock BJ, Matlaga BR. Validity of administrative coding in identifying patients with upper urinary tract calculi. J Urol. 2010; 184:190-2. [PubMed: 20478584]

27. Preminger GM, Tiselius H-G, Assimos DG, Alken P, Colin Buck A, Gallucci M, et al. 2007 guideline for the management of ureteral calculi. Eur Urol. 2007; 52:1610-31. [PubMed: 18074433] 
Children Presenting to ED

with Nephrolithiasis

( $N=2616)$

Children meeting coverage and

prescription inclusion criteria

( $N=1941)$

Excluded from analysis:

Stone Surgery at same visit ( $N=405$ )

Stone Surgery within 30 days prior to visit

$(N=19)$

Urinary Tract Infection ( $\mathrm{N}=232$ )

Acute Renal Insufficiency ( $\mathrm{N}=35$ )

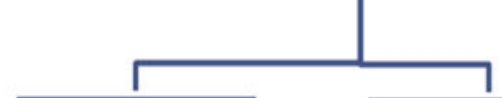

\section{(+) MET \\ $(\mathrm{N}=175)$}

(-) MET

$(\mathrm{N}=1150)$

CPT codes: Ureteral stent (52332); ESWL (950590); Ureteroscopy (52351, 52352, 52353, 52335, 52336, 52337), Nephrostomy $(50392,50393)$

Stone surgery at same visit: Nephrostomy (16), Ureteroscopy (220), ESWL (22), Ureteral stent (318)

Stone surgery w/in 30 days: Nephrostomy (4), Ureteroscopy (9), ESWL(0), Ureteral stent (13)

Figure 1.

Study flowchart of $n=1325$ participants. 


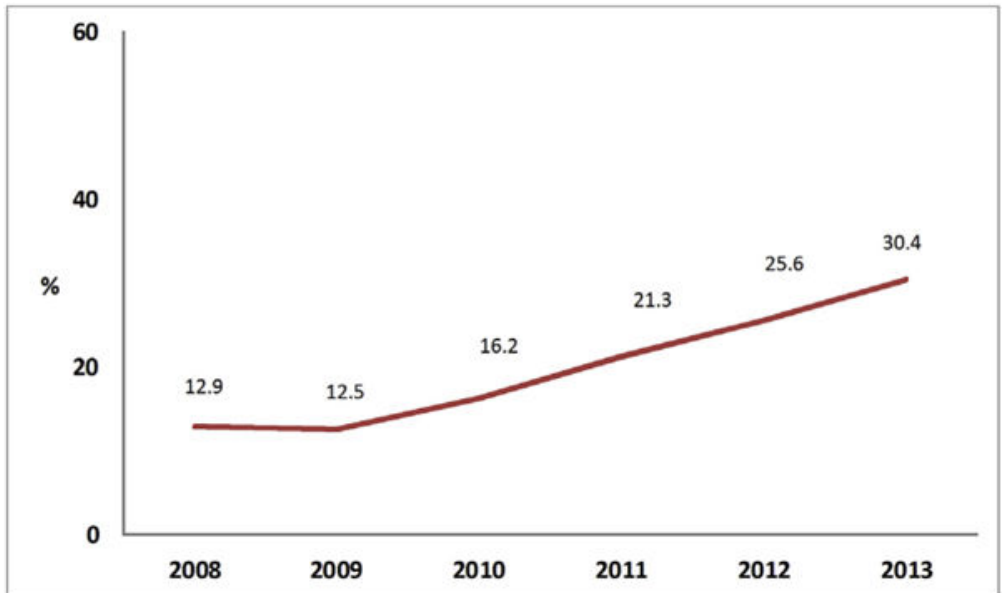

Figure 2.

Use of MET for ureteral calculi increases over study period $(p=0.001)$. 
Table

OR and CI for factors associated with use of MET.

\begin{tabular}{|c|c|c|}
\hline \multirow[t]{2}{*}{ Demographic } & Whole group $(n=1325)$ & Calculus of the ureter $(n=527)$ \\
\hline & OR $(95 \% \mathrm{CI})$ & OR $(95 \% \mathrm{CI})$ \\
\hline \multicolumn{3}{|c|}{ Factors associated with receipt of MET } \\
\hline Age & $1.05(1.00-1.10)$ & $1.02(0.95-1.10)$ \\
\hline Female & $0.64(0.46-0.90)$ & $0.77(0.47-1.28)$ \\
\hline Urologist provider & $2.04(1.32-3.15)$ & $1.60(0.88-2.90)$ \\
\hline Year of diagnosis & $1.31(1.19-1.44)$ & $1.34(1.15-1.56)$ \\
\hline \multicolumn{3}{|c|}{ Outcomes associated with receipt of MET } \\
\hline ED visit & $0.63(0.34-1.19)$ & $1.36(0.59-3.12)$ \\
\hline Hospital admission & $0.94(0.59-1.48)$ & $1.28(0.64-2.56)$ \\
\hline Appropriate imaging & $1.67(1.21-2.32)$ & $1.69(1.03-2.77)$ \\
\hline Surgical intervention & $1.11(0.77-1.60)$ & $0.96(0.57-1.63)$ \\
\hline
\end{tabular}




\section{Table 1}

Demographic comparison between patients with "calculus of the ureter" (ICD-09 592.1) and all other diagnoses of nephrolithiasis (ICD-09 592.0, 274.11, 592.9).

\begin{tabular}{|c|c|c|c|}
\hline Demographic & Calculus of ureter $(N=527)$ & All others $(N=798)$ & $p$-value \\
\hline Median age in years (range) & $16(1-18)$ & $16(1-18)$ & 0.33 \\
\hline Gender & & & 0.90 \\
\hline Male & $198(37.6 \%)$ & $297(37.2 \%)$ & \\
\hline Female & $329(62.4 \%)$ & $501(62.8 \%)$ & \\
\hline \multicolumn{4}{|l|}{ Comorbidity } \\
\hline Complex chronic condition & $62(11.8 \%)$ & $128(16.0 \%)$ & 0.03 \\
\hline Region & & & 0.002 \\
\hline Northeast & $74(14.0 \%)$ & $177(22.2 \%)$ & \\
\hline North Central & $136(25.8 \%)$ & $193(24.2 \%)$ & \\
\hline South & $239(45.4 \%)$ & $297(37.2 \%)$ & \\
\hline West & $68(12.9 \%)$ & $112(14.0 \%)$ & \\
\hline Unknown & $10(1.9 \%)$ & $19(2.4 \%)$ & \\
\hline Insurance status & $(\mathrm{n}=19$ missing $)$ & $(\mathrm{n}=47$ missing $)$ & 0.01 \\
\hline Comprehensive & $15(2.9 \%)$ & $13(1.7 \%)$ & \\
\hline EPO & $2(0.4 \%)$ & $22(2.9 \%)$ & \\
\hline HMO & $69(13.6 \%)$ & $131(17.4 \%)$ & \\
\hline POS & $36(7.1 \%)$ & $44(5.9 \%)$ & \\
\hline PPO & $357(70.3 \%)$ & $487(64.9 \%)$ & \\
\hline POS w/capitation & $4(0.8 \%)$ & $7(0.9 \%)$ & \\
\hline CDHP & $16(3.1 \%)$ & $32(4.3 \%)$ & \\
\hline HDHP & $9(1.8 \%)$ & $15(2.0 \%)$ & \\
\hline Received MET & & & 0.06 \\
\hline Yes & $81(15.4 \%)$ & $94(11.8 \%)$ & \\
\hline No & $446(84.6 \%)$ & $704(88.2 \%)$ & \\
\hline Type of MET & & & 1.00 \\
\hline Tamsulosin & $78(96.3 \%)$ & $88(93.6 \%)$ & \\
\hline Doxazosin & $2(2.5 \%)$ & $3(3.1 \%)$ & \\
\hline Alfuzosin & $1(1.2 \%)$ & $1(1.1 \%)$ & \\
\hline Terazosin & 0 & $1(1.1 \%)$ & \\
\hline Nifedipine & 0 & $1(1.1 \%)$ & \\
\hline \multicolumn{4}{|c|}{ Provider (categories not mutually exclusive) } \\
\hline Urologist & $98(18.6 \%)$ & $76(9.5 \%)$ & $<0.0001$ \\
\hline Nephrologist & $4(0.8 \%)$ & $7(0.9 \%)$ & 0.82 \\
\hline Pediatric urologist/nephrologist & $13(2.5 \%)$ & $9(1.1 \%)$ & 0.06 \\
\hline \multicolumn{4}{|l|}{ Time of ED visit to MET exposure } \\
\hline Days, mean (SD) & $1.78(1.34)$ & $1.76(1.46)$ & 0.92 \\
\hline
\end{tabular}

EPO, exclusive provider organization; HMO, health maintenance organization; POS, point of service; PPO, preferred provider organization; CDHP, consumer directed health plan; HDHP, high-deductible health plan; ED, emergency department; MET, medical expulsive therapy.

J Pediatr Urol. Author manuscript; available in PMC 2018 May 11. 


\section{Table 2}

Clinical predictors of MET for the entire cohort (A) and those patients with a diagnosis of "calculus of the ureter" (B).

\begin{tabular}{lll}
\hline Demographic & Unadjusted OR (95\% CI) & Adjusted OR (95\% CI $)^{\boldsymbol{a}}$ \\
\hline A) Whole group $(n=1325)$ & & \\
Age & $1.03(0.98-1.08)$ & $1.05(1.00-1.10)$ \\
Female & $0.71(0.51-0.98)$ & $0.67(0.48-0.94)$ \\
Urologist provider & $1.66(1.10-2.53)$ & $1.96(1.27-3.01)$ \\
Complex chronic condition & $1.16(0.75-1.80)$ & $1.06(0.68-1.67)$ \\
Year of diagnosis & $1.28(1.17-1.40)$ & $1.30(1.18-1.43)$ \\
B) Calculus of the ureter $(n=527)$ & \\
Age & $1.01(0.94-1.08)$ & $1.02(0.95-1.10)$ \\
Female & $0.80(0.50-1.30)$ & $0.79(0.48-1.30)$ \\
Urologist provider & $1.31(0.73-2.33)$ & $1.52(0.82-2.75)$ \\
Complex chronic condition & $0.93(0.44-1.96)$ & $0.82(0.38-1.79)$ \\
Year of diagnosis & $1.32(1.14-1.53)$ & $1.34(1.16-1.56)$ \\
\hline
\end{tabular}

${ }^{a}$ Adjusted for all other demographic variables in table. 
